\title{
Designing non-equilibrium states of quantum matter through stochastic resetting
}

\author{
Gabriele Perfetto, ${ }^{1}$ Federico Carollo, ${ }^{1}$ Matteo Magoni, ${ }^{1}$ and Igor Lesanovsky ${ }^{1,2}$ \\ ${ }^{1}$ Institut für Theoretische Physik, Eberhard Karls Universität Tübingen, \\ Auf der Morgenstelle 14, 72076 Tübingen, Germany. \\ ${ }^{2}$ School of Physics and Astronomy and Centre for the Mathematics \\ and Theoretical Physics of Quantum Non-Equilibrium Systems, \\ The University of Nottingham, Nottingham, NGr 2RD, United Kingdom.
}

\begin{abstract}
We consider closed quantum many-body systems subject to stochastic resetting. This means that their unitary time evolution is interrupted by resets at randomly selected times. When a reset takes place the system is reinitialized to a state chosen from a set of reset states conditionally on the outcome of a measurement taken immediately before resetting. We construct analytically the resulting non-equilibrium stationary state, thereby establishing a novel connection between quantum quenches in closed systems and the emergent open system dynamics induced by stochastic resetting. We discuss as an application the paradigmatic transverse-field quantum Ising chain. We show that signatures of its ground-state quantum phase transition are visible in the steady state of the reset dynamics as a sharp crossover. Our findings show that a controlled stochastic resetting dynamics allows to design non-equilibrium stationary states of quantum many-body systems, where uncontrolled dissipation and heating can be prevented. These states can thus be created on demand and exploited, e.g., as a resource for quantum enhanced sensing on quantum simulator platforms.
\end{abstract}

Introduction.- Deterministic or stochastic dynamics interspersed with stochastic resetting leads to the emergence of a non-equilibrium steady state (NESS). This concept has been put forward in the classical realm in Refs. [1-5], where it has been established that a particle undergoing Brownian motion reaches a NESS when its diffusive time evolution is reset at constant rate $\gamma$ (Poissonian reset) to its initial position. Using the renewal equation approach [1, 2, 6-13], it has been further shown that stochastic resetting can improve the efficiency of search processes in terms of their mean first passage time $[1,2,14-24]$. In contrast to the case of classical systems, much less is known about the effect of stochastic resetting on quantum systems, either closed or open. In the latter case, the Markovian Lindblad equation [25, 26] describing the evolution in the presence of Poissonian resetting has been analyzed in Refs. [27-32]. Closed quantum systems which are reinitialized to their initial state after Poissonian resetting have been, instead, analyzed without reference to the Lindblad equation in Ref. [33]. Using the renewal equation approach, it was shown in the latter work that the reached NESS differs from the diagonal ensemble for any finite, non-zero, value of $\gamma$. For $\gamma \rightarrow \infty$, instead, one recovers the quantum Zeno effect [34, 35], where the system does not evolve in time due to frequent reset projections. Resetting in closed quantum systems has been also studied in Refs. [36-41] for first detection problems. These recent works suggest that there is a direct link between the dynamics of closed quantum systems subject to stochastic resetting and an effective open system dynamics. However, the precise nature of this relation, and of possible connections between the NESS emerging from the resetting, unitary dynamics, and non-equilibrium signatures of quantum phase transitions [42-44] is not fully understood.
In this work we establish such a connection by developing a general theory based on semi-Markov processes $[30,45]$. We prove analytically that by averaging the microscopic unitary time evolution over the reset distribution an effective non-Markovian open dynamics, governed by a generalized Lindblad equation, emerges. The ensuing NESS can be constructed analytically and its structure establishes a transparent connection to quantum quenches in closed systems, which can display signatures of equilibrium quantum phase transitions [46-53]. Our results for the NESS recover the quantum Zeno effect in the limit of infinite resetting rate. We illustrate our ideas using, as an example, the quantum Ising chain in a transverse field (TFIC). The ground-state quantum phase transition of the model is signalled in the NESS by a crossover, whose sharpness is controlled by the distribution of the reset time. Our analysis establishes stochastic resetting as a tool to generate complex stationary many-body quantum states without detrimental processes, such as heating, that typically occur when coherent dynamics is competing with dissipative processes.

Reset protocol - The results we are going to develop in the following hold for a general many-body quantum system made of $N$ particles or spin degrees of freedom, but for concreteness we will base our discussion mostly on the paradigmatic TFIC with Hamiltonian

$$
H=-J \sum_{n=1}^{N}\left(\sigma_{n}^{x} \sigma_{n+1}^{x}+h \sigma_{n}^{z}\right) .
$$

Here $\sigma_{n}^{x, y, z}$ are the Pauli matrices acting on site $n, J>0$ is the ferromagnetic coupling constant and $h$ is the strength of a transverse magnetic field. This model exhibits a quantum phase transition in its ground state at the critical field strength $h=h_{c}=1$, which can be characterized by using the magnetization density $m=\sum_{n=1}^{N} \sigma_{n}^{x} / N$ as 
order parameter.

We envisage a "conditional reset" protocol, which is sketched in Fig. 1(a): Starting from a given initial state, in the Figure the state $\left.\left|\psi_{0}\right\rangle=|\uparrow \uparrow \ldots \uparrow\rangle[54]\right)$, the system is evolved unitarily under its many-body Hamiltonian $H$, e.g., Eq. (1) for the TFIC. After a randomly selected time, an observable is measured, in the Figure we measure the magnetization, and the many-body state is reset to a state chosen within a set of "reset states" conditionally on the outcome of the measurement. In our example the reset states are $|1\rangle=|\uparrow \uparrow \ldots \uparrow\rangle$ and $|2\rangle=|\downarrow \downarrow \ldots \downarrow\rangle$ (with $|\uparrow\rangle$ and $|\downarrow\rangle$ referring to the longitudinal- $x$ direction in Eq. (1)). Our motivation to investigate the two aforementioned reset states is twofold: first, they are simple product states easy to prepare experimentally; second, they allow to establish a connection between the NESS of the reset dynamics and quantum quenches in the Ising chain. The two reset states can be indeed identified with the two degenerate ground states $|\mathrm{GS}(h=0)\rangle_{ \pm}$of $H$ in Eq. (1) for $h=0$, namely, $|1\rangle=|\mathrm{GS}(h=0)\rangle_{+}$and $|2\rangle=|\operatorname{GS}(h=0)\rangle_{-}$. The unitary dynamics from the reset state $|j\rangle(j=1,2),\left|\psi_{j}(\tau)\right\rangle=\exp (-i H \tau)|j\rangle$ (setting $\hbar=1$ ), between consecutive resets stems therefore from a quench of the transverse field from the pre-quench $h_{0}=0$ to the post-quench value $h[46-53,55-63]$. The experimental motivation behind the conditional reset protocol comes from recent results on Rydberg quantum simulators [64], which allow to experimentally implement Ising models with transverse and longitudinal fields [65-72] and permit, at the same time, the spatially resolved detection of the spin state upon which the reset is conditioned. Such measurement has been indeed implemented in Rydberg atoms experiments on quantum quenches of the Ising model [73-76].

After the reset, the system evolves again under the quench dynamics up to the time at which the next measurement is performed. The time between consecutive measurements is drawn from a waiting time probability density, $p(\tau)$, which is normalized as $\int_{0}^{\infty} \mathrm{d} \tau p(\tau)=1$. Repeating this procedure leads at long times to a NESS, which in the case of the TFIC exhibits a sharp crossover between two stationary phases, as sketched in Fig. 1(b). Resetting can thus be interpreted as a dissipative process which leads to an effective open-system evolution.

The reset protocol also allows to suppress naturally occurring dissipative processes, e.g. spontaneous photon emission or scattering in cold atoms experiments. This is achieved by choosing the probability $p(\tau)$ such that the reset must occur before a finite maximum reset time $t_{\text {max }}$, which is shorter than the dissipation timescale. This may allow to reduce decoherence and heating and thus enables one to tailor states of matter with robustness and long-term stability. In the following we will develop the theory for deriving the form of this NESS and we discuss specifically the case of the TFIC. Note, however, that although the present discussion is based on two reset

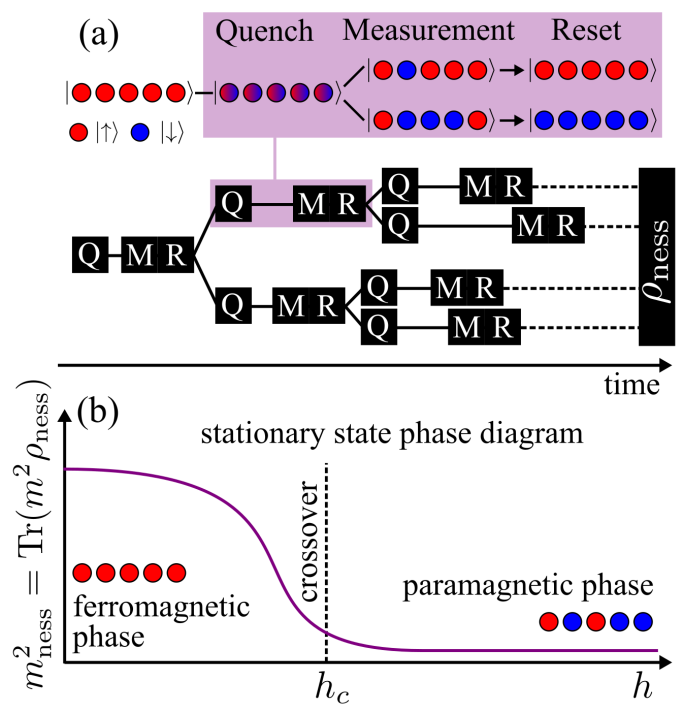

Figure 1. Conditional reset protocol. (a) The time evolution is obtained upon alternating the quench-unitary dynamics, schematized by the "Q" boxes, and measurement-reset events, schematized by the "M-R" boxes, which happen at randomly selected times from a waiting time distribution. The average over all the ensuing "reset trajectories" generates the NESS density matrix $\rho_{\text {ness. }}$. In the inset (purple rectangle) the time evolution ensuing in a single quench dynamics and reset is shown. Conditioned on the measured value of the magnetization being positive or negative, the system is reinitialized either in a reset state completely polarized upwards $(|1\rangle=|\uparrow \uparrow \ldots \uparrow\rangle)$ or downwards $(|2\rangle=|\downarrow \downarrow \ldots \downarrow\rangle)$, respectively. (b) Sketch of the squared magnetization density $m_{\text {ness }}^{2}$ in the NESS as a function of the transverse field $h$ [see Eqs. (1) and (9)]. It displays remnants of the equilibrium quantum phase transition at $h_{c}=1$ through a sharp crossover between a ferromagnetic and a paramagnetic stationary phase.

states, our derivations can be generalized to the case of an arbitrary number of reset states [77].

Non-equilibrium stationary state- When a reset takes place, the system is reinitialized in the reset state $|k\rangle$ $(k=1,2)$ dependently on the sign of the measured magnetization, see Fig. 1(a). Which reset state is chosen depends only on the time $\tau$ elapsed since the previous reset event and on its outcome $|j\rangle(j=1,2)$, but, fundamentally, not on the previous history of the dynamics. The conditional reset dynamics can be therefore understood as a semi-Markov process [30, 45], which is characterized by the transition probabilities $P_{j k}(\tau)$ (obeying $\left.\sum_{k} P_{j k}(\tau)=1\right)$ from the previous reset state $|j\rangle$ to the following reset state $|k\rangle$, given that a time $\tau$ has elapsed between the two resets. The formalism of semi-Markov processes has been applied in Ref. [30] to Markovian open quantum system. Here we consider, instead, the case of an underlying unitary time evolution, and for the conditional reset dynamics sketched in Fig. 1 we write

$$
P_{j k}(\tau)=\left\langle\psi_{j}(\tau)\left|\mathcal{P}_{k}\right| \psi_{j}(\tau)\right\rangle
$$


where $\mathcal{P}_{k}$ denotes the projector onto the set of states of the Hilbert space with positive $(k=1)$ or negative $(k=2)$ magnetization [78].

To describe the time evolution of the density matrix $\rho(t)$ we exploit the fact that the resetting protocol induces a renewal structure, in the sense that the dynamics after a certain reset does not depend on what happened before that reset. This observation allows to derive "renewal equations" which express the dynamics of the state $\rho(t)$ in the presence of resets, in terms of the state $\rho_{\text {free }, j}(t)=\left|\psi_{j}(t)\right\rangle\left\langle\psi_{j}(t)\right|$ which evolves under a dynamics where resets are absent. We stress that in our analysis the resetting is coupled to the underlying unitary dynamics via Eq. (2). This makes the conditional reset dynamics fundamentally different with respect to resets to a single state, first analyzed in Ref. [33] for closed quantum systems, where the reset is independent from the underlying dynamics, which significantly simplifies the analysis.

We consider the observation time $t$ to be fixed and calculate the average density matrix $\rho_{n}(t)$ over all the "reset trajectories" that have exactly $n \geq 0$ resets within the interval $[0, t]$. The density matrix is then given by accounting for all the reset trajectories with an arbitrary number of resets $\rho(t)=\sum_{n=0}^{\infty} \rho_{n}(t)$, as shown pictorially in Fig. 1(a). The previous expression can be resummed by taking the Laplace transform $\widehat{\rho}(s)$ of $\rho(t)$. The average density matrix $\widehat{\rho}_{n}(s)$ is, indeed, obtained starting from the initial state, similarly as in Markov chains, by considering $n$ reset transitions $\widehat{R}^{n}(s)$, with the transition matrix $\widehat{R}_{j k}(s)$ :

$$
\widehat{R}_{j k}(s)=\int_{0}^{\infty} \mathrm{d} \tau P_{j k}(\tau) p(\tau) e^{-s \tau} .
$$

In the previous equation, $R_{j k}(\tau)=P_{j k}(\tau) p(\tau)$ is the probability density that the system is reinitialized in the reset state $|k\rangle$ in the time interval $(\tau, \tau+\mathrm{d} \tau)$ since the previous reset to the reset state $|j\rangle$. Note that $\widehat{R}_{j k}(s=0)$ is a Markov matrix since $\sum_{k} \widehat{R}_{j k}(s=0)=1$. The resulting expression for $\widehat{\rho}(s)$ is [77]

$$
\widehat{\rho}(s)=\widehat{\rho}_{01}(s)+\widehat{\rho}_{01}(s) \widehat{r}_{11}(s)+\widehat{\rho}_{02}(s) \widehat{r}_{12}(s),
$$

with

$$
\widehat{\rho}_{01}(s)=\int_{0}^{\infty} \mathrm{d} \tau q(\tau) \rho_{\text {free }, 1}(\tau) e^{-s \tau},
$$

and analogously for $\widehat{\rho}_{02}(s)$ in terms of $\rho_{\text {free }, 2}(t)$. In Eq. (5) we have introduced the survival probability $q(\tau)=$ $\int_{\tau}^{\infty} \mathrm{d} \tau^{\prime} p\left(\tau^{\prime}\right)$, which is the probability that no reset happens before time $\tau$.

The case of Poissonian resetting with constant rate $\gamma$ corresponds to $p(\tau)=p_{\gamma}(\tau)=\gamma \exp (-\gamma \tau)$ and $q(\tau)=$ $q_{\gamma}(\tau)=\exp (-\gamma \tau)$. The derivations in this manuscript apply, however, to arbitrary distributions $p(\tau)$.

The first term in Eq. (4), involving $\widehat{\rho}_{01}(s)$, accounts for trajectories where no reset takes place and it is therefore given by the unitary time evolution from the initial state $\left|\psi_{0}\right\rangle=|1\rangle$ over the interval $[0, t]$. The coefficients $r_{11}(t) \geq 0$ and $r_{12}(t) \geq 0$, which are obtained from the inverse Laplace transform of $\widehat{r}_{11}(s)=\sum_{n=1}^{\infty}\left(\widehat{R}^{n}(s)\right)_{11}$ and $\widehat{r}_{12}(s)=\sum_{n=1}^{\infty}\left(\widehat{R}^{n}(s)\right)_{12}$ in Eq. (4), can be interpreted as two effective time-dependent rates of jumping into the reset states $|1\rangle$ and $|2\rangle$, respectively. Crucially, $r_{11}(t)$ and $r_{12}(t)$ are determined both by the resetting distribution $p(\tau)$ and by the Hamiltonian dynamics, which are coupled due to the conditional protocol. The calculation of the NESS density matrix $\rho_{\text {ness }}$ can then be directly performed from Eq.(4) as $\rho_{\text {ness }}=\lim _{t \rightarrow \infty} \rho(t)=\lim _{s \rightarrow 0} s \widehat{\rho}(s)$, which gives:

$$
\begin{aligned}
\rho_{\text {ness }} & =\frac{\widehat{R}_{21}(0)}{\widehat{R}_{21}(0)+\widehat{R}_{12}(0)} \frac{1}{\widehat{q}(0)} \int_{0}^{\infty} \mathrm{d} \tau \rho_{\text {free }, 1}(\tau) q(\tau) \\
& +\frac{\widehat{R}_{12}(0)}{\widehat{R}_{21}(0)+\widehat{R}_{12}(0)} \frac{1}{\widehat{q}(0)} \int_{0}^{\infty} \mathrm{d} \tau \rho_{\text {free }, 2}(\tau) q(\tau) .
\end{aligned}
$$

This equation is the first main result of this work. It expresses $\rho_{\text {ness }}$ as a statistical mixture of the unitary (reset-free) time evolution ensuing from the reset states $|1\rangle$ and $|2\rangle$. Fundamentally, both the weights of the terms in the sum couple the Hamiltonian dynamics with the reset via Eqs. (2) and (3). One can further check [77], as noted also in Ref. [33] for resets to a single state, that $\rho_{\text {ness }}$ has non-vanishing off-diagonal matrix elements in the basis of the eigenstates of the Hamiltonian $H$. This shows that $\rho_{\text {ness }}$ is a genuinely non-equilibrium density matrix since it is different from the diagonal ensemble, which describes the relaxation at long times of closed quantum systems [33, 79].

A complementary way to the renewal equation approach, is to construct an evolution equation for $\rho(t)$, which for classical systems has been done in Ref. [5]. This perspective is useful as it sheds light on the effective open system dynamics which emerges upon superimposing resetting to the unitary time evolution of the system. For our conditional reset protocol we find [77] from Eq. (4) the generalized Lindblad equation

$$
\begin{aligned}
\frac{\mathrm{d} \rho(t)}{\mathrm{d} t}= & \mathcal{L}[\rho(t)]+r_{11}(t)|1\rangle\left\langle 1\left|+r_{12}(t)\right| 2\right\rangle\langle 2| \\
& -\int_{0}^{t} \mathrm{~d} t^{\prime} r\left(t-t^{\prime}\right) e^{\left(t-t^{\prime}\right) \mathcal{L}} \rho\left(t^{\prime}\right),
\end{aligned}
$$

with the time-dependent rates $r_{1 k}(t)$, with $k=1,2$, written in terms of the density matrix [77] as

$$
r_{1 k}(t)=\int_{0}^{t} \mathrm{~d} t^{\prime} r\left(t-t^{\prime}\right) \operatorname{Tr}\left[\mathcal{P}_{k} e^{\left(t-t^{\prime}\right) \mathcal{L}} \rho\left(t^{\prime}\right)\right] .
$$

In Eq. (7), $\mathcal{L}[\rho]=-i[H, \rho]$ is the generator of the unitary time evolution and $r(t)$ satisfies $r_{11}(t)+r_{12}(t)=$ $\int_{0}^{t} \mathrm{~d} t^{\prime} r\left(t^{\prime}\right)$, which ensures a trace-preserving dynamics.

Equations (7) and (8) are our second main result. They show that stochastic resetting is generating an engineered 


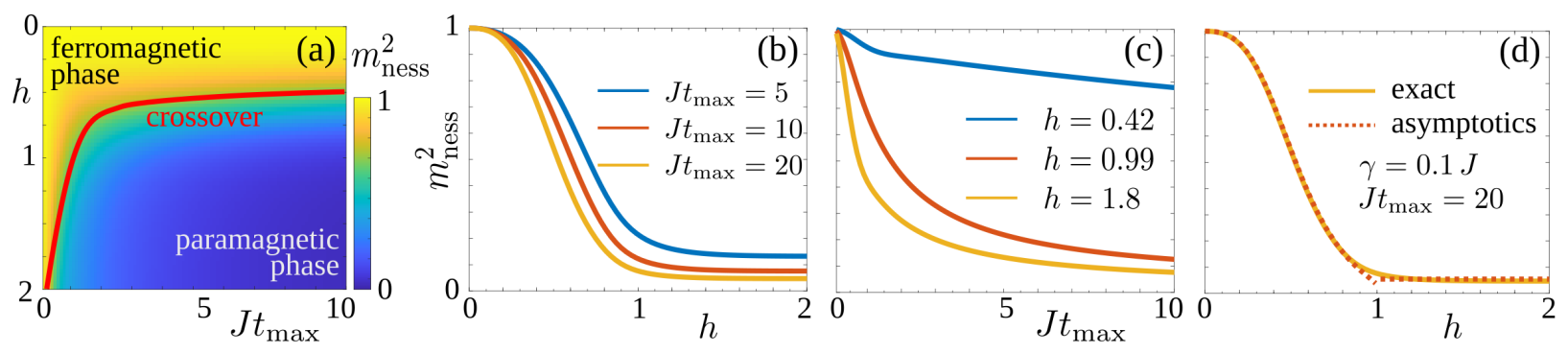

Figure 2. NESS phase diagram. (a) Squared magnetization density, $m_{\text {ness }}^{2}$, in the TFIC as a function of transverse field $h$ and (scaled) maximum reset time $J t_{\max }$. The red line is a guide for the eye showing the region where the crossover from the ferromagnetic to the paramagnetic stationary phase takes place. (b) Plot of $m_{\text {ness }}^{2}$ as a function of $h$ for fixed values of $J t_{\max }=5,10,20$ (from top to bottom). As the maximum reset time increases the crossover becomes sharper and resembles more and more the equilibrium quantum phase transition behavior. (c) Plot of $m_{\text {ness }}^{2}$ as a function of $J t_{\text {max }}$ for different fixed values of $h=0.42,0.99,1.8$ (from top to bottom). (d) Comparison between the result for $m_{\text {ness }}^{2}$ as a function of $h$ from the exact evaluation of Eq. (9) with $\left\langle\sigma^{x}(\tau)\right\rangle$ obtained from the clustering property of the two-point function (yellow solid line), and the result obtained using the asymptotic expression of $\left\langle\sigma^{x}(\tau)\right\rangle$ valid for large $\tau$ (red dashed line). In the Figure $\gamma=0.1 J$.

dissipative process which leads to an emergent open dynamics for the system without an actual environment being present. The dynamics in Eqs. (7) and (8) is in general non-Markovian because of the presence of the integral over the complete time history of the process. This is a consequence, as in the classical case of Ref. [5], of the fact that for non-Poissonian distributions $p(\tau)$ one has to keep track of the time elapsed since the last reset. Only in the Poissonian case, $p_{\gamma}(\tau)$, resetting occurs at constant rate $\gamma$, independently of the time elapsed since the last reset. In this case one verifies that $r(t)=\gamma \delta(t)$ in Eq. (7), which gives back a Markovian-Lindblad dynamics [27-29, 31, 32, 77].

The quantum Ising chain in a transverse field-We particularize in this Section our theory to the TFIC introduced above [see Eq. (1)]. Periodic boundary conditions will be henceforth assumed. The quantum critical point at $h=h_{c}=1$ separates [80-83], at zero temperature and in the thermodynamic limit $N \rightarrow \infty$, the paramagnetic $(h>1)$ from the ferromagnetic $(h<1)$ phase. In the latter, because of the spontaneous breaking of the $\mathbb{Z}_{2}$ symmetry, the ground-state expectation value of the magnetization density $m$ becomes non-zero ${ }_{ \pm}\langle\operatorname{GS}(h)|m| \operatorname{GS}(h)\rangle_{ \pm}= \pm\left(1-h^{2}\right)^{1 / 8}$, where $|\operatorname{GS}(h)\rangle_{ \pm}$ are the two degenerate ground states for $h<1$.

Because of the $\mathbb{Z}_{2}$ symmetry, the matrices $P_{j k}(\tau)$ and $\widehat{R}_{j k}(s)$ in Eqs. (2) and (3) are symmetric. This, in turn, implies that the magnetization density in the NESS in Eq. (6) is identically zero: $\operatorname{Tr}\left(m \rho_{\text {ness }}\right)=0$ (and the same for all the odd operators under the $\mathbb{Z}_{2}$ symmetry [77]). The natural order parameter to distinguish between the ordered and the disordered phase is then the squared magnetization density $m_{\text {ness }}^{2}=\operatorname{Tr}\left(m^{2} \rho_{\text {ness }}\right)$. For the latter one can derive, in the thermodynamic limit $N \rightarrow \infty$, the expression [77]

$$
m_{\text {ness }}^{2}=\frac{1}{\widehat{q}(0)} \int_{0}^{\infty} \mathrm{d} \tau q(\tau)\left\langle\sigma^{x}(\tau)\right\rangle^{2},
$$

which directly connects the NESS expectation value of $m_{\text {ness }}^{2}$ in the presence of reset with the magnetization density quench dynamics $\langle m(\tau)\rangle=\left\langle\sigma^{x}(\tau)\right\rangle$ (with the last equality coming from the choice of periodic boundary conditions). Equation (9) can be evaluated exactly in the thermodynamic limit upon extracting $\left\langle\sigma^{x}(\tau)\right\rangle$ from the cluster decomposition principle of the twopoint function $\left\langle\sigma_{n}^{x} \sigma_{n+l}^{x}\right\rangle(\tau)$ at large separation $l$ [77], as outlined first in [84-87] for the equilibrium correlation function and in [49-51] for the non-equilibrium quench dynamics. In the latter case, it is known [49-51, 63] that $\left\langle\sigma^{x}(\tau)\right\rangle \propto \exp \left(-t \Lambda\left(h, h_{0}\right)\right)$ relaxes exponentially to zero at long times with a characteristic time scale $\Lambda\left(h, h_{0}\right)^{-1}>0$ set by the quench [77].

In Fig. 2 we evaluate Eq. (9) for the waiting time distribution $p(\tau)=p_{\gamma, t_{\max }}(\tau)=\gamma e^{-\gamma \tau} /\left(1-e^{-\gamma t_{\max }}\right) \Theta\left(t_{\max }-\right.$ $\tau)$, which represents a "chopped Poissonian distribution" with rate $\gamma$ and maximum reset time $t_{\max }[\Theta(\tau)$ denotes the Heaviside step function]. The NESS reflects the equilibrium quantum phase transition through a sharp crossover due to the competition between the maximum reset time $t_{\max }$ and the order parameter decay time $\Lambda\left(h, h_{0}\right)^{-1}$. Upon increasing $J t_{\max }$ with fixed $\gamma / J$ (or, vice-versa, decreasing $\gamma / J$ with $J t_{\max }$ fixed) the crossover becomes very sharp, as shown in Fig. 2(b) for $\gamma=0.1 J$ and $J t_{\max }=20$. We emphasize that $m_{\text {ness }}^{2}$ is always different from zero, even for large values of $h$ deep in the paramagnetic phase, for any finite value of $J t_{\max }$ or $\gamma / J$. This means that $\rho_{\text {ness }}$, thanks to the resetting protocol, retains part of the long-range order stored in the reset states $|1\rangle$ and $|2\rangle$. For the reset-free quench time evolution, indeed, the diagonal ensemble predicts a 
vanishing expectation value of $m^{2}$ and therefore a complete loss of the order of $|1\rangle$ and $|2\rangle$. As $J t_{\max } \rightarrow 0$ one encounters the quantum Zeno effect $[34,35]$ since the initial state does not evolve in time due to very frequent resets and therefore the squared magnetization density remains close to 1 . In the limit of large $J t_{\max }(\operatorname{small} \gamma / J)$, when the crossover gets sharp and it closely resembles the equilibrium quantum phase transition, a large time, on average, elapses between consecutive resets. As a consequence, one can evaluate Eq. (9) using the asymptotics of $\left\langle\sigma^{x}(\tau)\right\rangle \propto \exp \left(-t \Lambda\left(h, h_{0}\right)\right)$ for long times $\tau \rightarrow \infty$, which has been analytically derived in Refs. [49-51, 77]. The result is shown in Fig. 2(d) [77]. We can see that the asymptotics of the order parameter is in excellent agreement with the exact numerical evaluation of Eq. (9). Only for values of $h \gtrsim 0.9$ discrepancies are visible. This is caused by the fact that when $h$ becomes large the order parameter $\left\langle\sigma^{x}(\tau)\right\rangle$ quickly relaxes to zero as a function of $\tau$ and the integral in Eq. (9) is therefore no longer dominated by the behavior of $\left\langle\sigma^{x}(\tau)\right\rangle$ at large times.

Outlook.- We have presented a general protocol for constructing the NESS of a unitary many-body dynamics interspersed by random resets. Using the TFIC as exemplary case, we have shown that this NESS is intimately connected to the dynamics following a quantum quench, and that it embodies the ground-state quantum phase transition of the TFIC through a sharp crossover. NESS with sharp crossovers can here be exploited to design collectively enhanced sensing protocols where a small change of the parameter (external perturbation) driving the quantum phase transition translates into an easy-to-detect macroscopic response, as, e.g., discussed in Refs. [88, 89]. A possible advantage of our protocol is, however, that the establishment of the NESS relies on stochastic resetting, which diminishes the impact of uncontrolled dissipative effects such as heating.

Acknowledgements.-We acknowledge support from the "Wissenschaftler Rückkehrprogramm GSO/CZS" of the Carl-Zeiss-Stiftung and the German Scholars Organization e.V., from the European Union's Horizon 2020 research and innovation program under grant agreement No. 800942 (ErBeStA), as well as from the BadenWürttemberg Stiftung through project BWST_ISF2019023.

[1] M. R. Evans and S. N. Majumdar, Phys. Rev. Lett. 106, 160601 (2011).

[2] M. R. Evans and S. N. Majumdar, J. Phys. A: Math. Theor. 44, 435001 (2011).

[3] M. R. Evans and S. N. Majumdar, J. Phys. A: Math. Theor. 47, 285001 (2014).

[4] S. N. Majumdar, S. Sabhapandit, and G. Schehr, Phys. Rev. E 91, 052131 (2015).

[5] S. Eule and J. J. Metzger, New J. Phys. 18, 033006 (2016).
[6] A. Pal, A. Kundu, and M. R. Evans, J. Phys. A: Math. Theor. 49, 225001 (2016).

[7] A. Pal and S. Reuveni, Phys. Rev. Lett. 118, 030603 (2017).

[8] A. Chechkin and I. M. Sokolov, Phys. Rev. Lett. 121, 050601 (2018).

[9] M. R. Evans and S. N. Majumdar, J. Phys. A: Math. Theor. 51, 475003 (2018).

[10] J. Masoliver and M. Montero, Phys. Rev. E 100, 042103 (2019).

[11] A. S. Bodrova, A. V. Chechkin, and I. M. Sokolov, Phys. Rev. E 100, 012119 (2019).

[12] A. S. Bodrova, A. V. Chechkin, and I. M. Sokolov, Phys. Rev. E 100, 012120 (2019).

[13] M. Magoni, S. N. Majumdar, and G. Schehr, Phys. Rev. Research 2, 033182 (2020).

[14] S. Redner, A guide to first-passage processes (Cambridge University Press, 2001).

[15] A. J. Bray, S. N. Majumdar, and G. Schehr, Adv. Phys. 62, 225 (2013).

[16] M. R. Evans, S. N. Majumdar, and K. Mallick, J. Phys. A: Math. Theor. 46, 185001 (2013).

[17] M. Montero and J. Villarroel, Phys. Rev. E 87, 012116 (2013).

[18] C. Christou and A. Schadschneider, J. Phys. A: Math. Theor. 48, 285003 (2015).

[19] D. Campos and V. m. c. Méndez, Phys. Rev. E 92, 062115 (2015).

[20] M. Montero, A. Masó-Puigdellosas, and J. Villarroel, The European Physical Journal B 90, 1 (2017).

[21] A. Masó-Puigdellosas, D. Campos, and V. m. c. Méndez, Phys. Rev. E 99, 012141 (2019).

[22] G. Tucci, A. Gambassi, S. Gupta, and E. Roldán, Phys. Rev. Res. 2, 043138 (2020).

[23] M. R. Evans, S. N. Majumdar, and G. Schehr, J. Phys. A: Math. Theor. 53, 193001 (2020).

[24] D. M. Busiello, D. Gupta, and A. Maritan, arXiv:2106.08044 (2021).

[25] G. Lindblad, Commun. Math. Phys. 48, 119 (1976).

[26] V. Gorini, A. Kossakowski, and E. C. G. Sudarshan, J. Math. Phys. 17, 821 (1976).

[27] L. Hartmann, W. Dür, and H.-J. Briegel, Phys. Rev. A 74, 052304 (2006).

[28] N. Linden, S. Popescu, and P. Skrzypczyk, Phys. Rev. Lett. 105, 130401 (2010).

[29] D. C. Rose, H. Touchette, I. Lesanovsky, and J. P. Garrahan, Phys. Rev. E 98, 022129 (2018).

[30] F. Carollo, R. L. Jack, and J. P. Garrahan, Phys. Rev. Lett. 122, 130605 (2019).

[31] A. Tavakoli, G. Haack, N. Brunner, and J. B. Brask, Phys. Rev. A 101, 012315 (2020).

[32] A. Riera-Campeny, J. Ollé, and A. Masó-Puigdellosas, arXiv:2011.04403 (2020).

[33] B. Mukherjee, K. Sengupta, and S. N. Majumdar, Phys. Rev. B 98, 104309 (2018).

[34] B. Misra and E. G. Sudarshan, J. Math. Phys. 18, 756 (1977).

[35] C. B. Chiu, E. C. G. Sudarshan, and B. Misra, Phys. Rev. D 16, 520 (1977).

[36] Y. Aharonov, L. Davidovich, and N. Zagury, Phys. Rev. A 48, 1687 (1993).

[37] D. A. Meyer, J. Stat. Phys. 85, 551 (1996).

[38] H. Friedman, D. Kessler, and E. Barkai, J. Phys. A: Math. Theor. 50, 04LT01 (2016). 
[39] H. Friedman, D. A. Kessler, and E. Barkai, Phys. Rev. E 95, 032141 (2017).

[40] F. Thiel, E. Barkai, and D. A. Kessler, Phys. Rev. Lett. 120, 040502 (2018).

[41] F. Thiel, I. Mualem, D. A. Kessler, and E. Barkai, Phys. Rev. Res. 2, 023392 (2020).

[42] S. L. Sondhi, S. M. Girvin, J. P. Carini, and D. Shahar, Rev. Mod. Phys. 69, 315 (1997).

[43] M. Vojta, Rep. Prog. Phys. 66, 2069 (2003).

[44] S. Sachdev, Quantum phase transitions (Cambridge University Press, 2011).

[45] J. Janssen and R. Manca, Applied semi-Markov processes (Springer Science \& Business Media, 2006).

[46] K. Sengupta, S. Powell, and S. Sachdev, Phys. Rev. A 69, 053616 (2004).

[47] P. Calabrese and J. Cardy, Phys. Rev. Lett. 96, 136801 (2006).

[48] P. Calabrese and J. Cardy, J. Stat. Mech.: Theory Exp. 2007, P06008 (2007).

[49] P. Calabrese, F. H. L. Essler, and M. Fagotti, Phys. Rev. Lett. 106, 227203 (2011).

[50] P. Calabrese, F. H. Essler, and M. Fagotti, J. Stat. Mech.: Theory Exp. 2012, P07016 (2012).

[51] P. Calabrese, F. H. Essler, and M. Fagotti, J. Stat. Mech.: Theory Exp. 2012, P07022 (2012).

[52] M. Heyl, A. Polkovnikov, and S. Kehrein, Phys. Rev. Lett. 110, 135704 (2013).

[53] M. Heyl, Rep. Prog. Phys. 81, 054001 (2018).

[54] The NESS does not depend on the specific choice of the initial state. The latter influences only the transient dynamics.

[55] F. H. Essler and M. Fagotti, J. Stat. Mech.: Theory Exp. 2016, 064002 (2016).

[56] F. Iglói and H. Rieger, Phys. Rev. Lett. 106, 035701 (2011).

[57] H. Rieger and F. Iglói, Phys. Rev. B 84, 165117 (2011).

[58] A. Silva, Phys. Rev. Lett. 101, 120603 (2008).

[59] F. H. L. Essler, S. Evangelisti, and M. Fagotti, Phys. Rev. Lett. 109, 247206 (2012).

[60] L. Foini, L. F. Cugliandolo, and A. Gambassi, J. Stat. Mech.: Theory Exp. 2012, P09011 (2012).

[61] F. Meinert, M. J. Mark, E. Kirilov, K. Lauber, P. Weinmann, A. J. Daley, and H.-C. Nägerl, Phys. Rev. Lett. 111, 053003 (2013).

[62] L. Bucciantini, M. Kormos, and P. Calabrese, J. Phys. A: Math. Theor. 47, 175002 (2014).

[63] C. B. Dağ and K. Sun, Phys. Rev. B 103, 214402 (2021).

[64] A. Browaeys and T. Lahaye, Nat. Phys. 16, 132 (2020).

[65] M. Kormos, M. Collura, G. Takács, and P. Calabrese, Nat. Phys. 13, 246 (2017).
[66] A. J. A. James, R. M. Konik, and N. J. Robinson, Phys. Rev. Lett. 122, 130603 (2019).

[67] N. J. Robinson, A. J. A. James, and R. M. Konik, Phys. Rev. B 99, 195108 (2019).

[68] P. P. Mazza, G. Perfetto, A. Lerose, M. Collura, and A. Gambassi, Phys. Rev. B 99, 180302 (2019).

[69] A. Lerose, F. M. Surace, P. P. Mazza, G. Perfetto, M. Collura, and A. Gambassi, Phys. Rev. B 102, 041118 (2020).

[70] G. Lagnese, F. M. Surace, M. Kormos, and P. Calabrese, J. Stat. Mech.: Theory Exp. 2020, 093106 (2020).

[71] R. Verdel, F. Liu, S. Whitsitt, A. V. Gorshkov, and M. Heyl, Phys. Rev. B 102, 014308 (2020).

[72] F. B. Ramos, M. Lencsés, J. C. Xavier, and R. G. Pereira, Phys. Rev. B 102, 014426 (2020).

[73] J. F. Sherson, C. Weitenberg, M. Endres, M. Cheneau, I. Bloch, and S. Kuhr, Nature 467, 68 (2010).

[74] P. Schauß, M. Cheneau, M. Endres, T. Fukuhara, S. Hild, A. Omran, T. Pohl, C. Gross, S. Kuhr, and I. Bloch, Nature 491, 87 (2012).

[75] J. Zeiher, P. Schauß, S. Hild, T. Macrì, I. Bloch, and C. Gross, Phys. Rev. X 5, 031015 (2015).

[76] H. Labuhn, D. Barredo, S. Ravets, S. De Léséleuc, T. Macrì, T. Lahaye, and A. Browaeys, Nature 534, 667 (2016).

[77] See the Supplemental Material for the details of the calculations.

[78] For an even number $N$ of spins, $\mathcal{P}_{k}$ is not a projector since one has to account for states with zero total magnetization. This does not spoil our general theory since the fundamental property $\sum_{k} P_{j k}(\tau)=1$ still holds [77].

[79] L. D'Alessio, Y. Kafri, A. Polkovnikov, and M. Rigol, Adv. Phys. 65, 239 (2016).

[80] E. Lieb, T. Schultz, and D. Mattis, Ann. Phys. 16, 407 (1961).

[81] S. Pikin and V. Tsukernik, J. Exp. Theor. Phys 23 (1966).

[82] P. Pfeuty, Ann. Phys. 57, 79 (1970).

[83] J. B. Kogut, Rev. Mod. Phys. 51, 659 (1979).

[84] E. Barouch, B. M. McCoy, and M. Dresden, Phys. Rev. A 2, 1075 (1970).

[85] E. Barouch and B. M. McCoy, Phys. Rev. A 3, 786 (1971).

[86] E. Barouch and B. M. McCoy, Phys. Rev. A 3, 2137 (1971).

[87] B. M. McCoy, E. Barouch, and D. B. Abraham, Phys. Rev. A 4, 2331 (1971).

[88] M. Raghunandan, J. Wrachtrup, and H. Weimer, Phys. Rev. Lett. 120, 150501 (2018).

[89] C. G. Wade, M. Marcuzzi, E. Levi, J. M. Kondo, I. Lesanovsky, C. S. Adams, and K. J. Weatherill, Nat. Commun. 9, 1 (2018). 